\title{
THE RELATIONSHIP BETWEEN URIC ACID LEVELS AND GRAFT FUNCTION IN RENAL TRANSPLANT PATIENTS WHO DISCONTINUED STEROID THERAPY
}

\author{
Hulya Colak ${ }^{1}$, Sibel Ersan ${ }^{2}$, Mehmet Tanrisev $^{2}$, Banu Yilmaz $^{2}$, Orcun Ural ${ }^{2}$, Zumrut \\ $\mathrm{Unal}^{3}$, and İsmail Sert ${ }^{2}$ \\ ${ }^{1}$ Affiliation not available \\ ${ }^{2}$ Republic of Turkey Ministry of Health Izmir Provincial Health Directorate Izmir \\ University of Health Sciences Tepecik Training and Research Hospital \\ ${ }^{3}$ Ege University Department of Textile Engineering
}

May 8, 2021

\begin{abstract}
Introduction: High uric acid levels are commonly encountered in kidney transplant recipients, and can be associated with allograft dysfunction. Our study aims to examine the relationship between UA levels and graft function in patients discontinuing steroids. Methods: In this single-center-retrospective study, 56 patients from among 678 RT patients transplanted from living donors between 1999-2020 were included. Causes of steroid discontinuation, creatinine levels concurrent with uric acid levels before and after steroid discontinuation (mean $3.9 \pm 2.1$ years), acute rejection numbers, demographics, durations of dialysis and transplantation, medications (d.a. use of immunosuppressive, antihypertensive), laboratory data, human leukocyte antigen (HLA) mismatch numbers, blood-pressure [BP], body mass index, late acute rejection (LAR) numbers (3 months post-transplantation) were all recorded. Results: Creatinine and uric acid levels were seen to have increased after steroid discontinuation, there was a significant relationship between them $(\mathrm{p}<0.001)$. Statistically significant correlation was found between increased creatinine levels after steroid discontinuation and graft survival with higher HLA mismatch; 39 (69.6\%) patients with mismatch [?]2, and 17 patients with mismatch $<2(30.4 \%)(\mathrm{p}=0.049)$. No significant relationship was found between LAR numbers before and after steroid discontinuation, and creatinine levels after steroid discontinuation. In conclusion, per model obtained as a result of multivariate linear analysis, hyperuricemia and HLA mismatch numbers $(\mathrm{p}=0.048$ and $\mathrm{p}=0.044$, respectively) are independent predictive factors for graft dysfunction in patients discontinuing steroids. Accordingly, negative effects of modeling should be kept in mind for long-term graft survival in patients who plan to continue with steroid-sparing regimens.
\end{abstract}

\section{INTRODUCTION:}

The relation between high serum uric acid levels and renal, cardiovascular, and metabolic disturbances since the 19 th century. ${ }^{1}$

Several epidemiological studies substanyially suggested that chronic hyperuricemia was an independent risk factor for hypertension, CVD, and chronic kidney disease. ${ }^{2,3,4}$ Therefore it is crucial to consider serum uric acid levels when profiling patient. The evidence suggests that serum uric acid levels above $6 \mathrm{mg} / \mathrm{dl}$ is considered abnormal..$^{5}$ Overall, hyperuricemia can induce renal and cardiovascular injury both directly and indirectly through well-known mechanisms of tissue damage. ${ }^{6}$ Several studies have reported that elevated serum UA levels correspond with reduced kidney transplant survival. ${ }^{3,7}$

Immunosuppressive agents initiated after renal transplantation (RT) have variable effects on 
serum UA levels. ${ }^{8}$ Previous studies have shown that steroids increase UA excretion. ${ }^{9}$

The aim of our study is to examine the relationship between UA levels and graft function in patients who discontinued steroids.

\section{Material and Method}

\section{Study Population}

In this study, planned as a single center (Tepecik Training and Research Hospital, The Renal Transplant Program, Izmir, Turkey) and retrospectively, 56 patients who underwent kidney transplantation from living donors $(\mathrm{n}=678)$ between 1999-2020 and who did not receive steroids were included. The average age of our study group [41 (73.2\%) males and 15 (26.8\%) females] was $45.8 \pm 8.8$ years.

In our patients, the reasons for not using steroids were osteoporosis $(\mathrm{n}=9 ; 16.1 \%)$, avascular necrosis of the femoral head $(n=6 ; 10.7 \%)$, dermatological reasons $(n=1 ; 1.2 \%)$ and unknown reasons for not taking steroids $(n=40 ; 71.5 \%)$. Exclusion criteria included inability to provide consent, pregnancy, New York Heart Association class III or IV congestive heart failure, gout disease, history of kidney stones , metabolic syndrome, human immunodeficiency virus infection, multiple myeloma, renal cancer, smoking, cirrhosis, history of malignancy, history of end-stage renal disease, proteinuria, being under 18, allopurinol use, diabetes mellitus, obesity, inflammation, history of ischemic coronary disease, uncontrolled hypertension, secondary hypertension, Cytomegalovirus and Polyomavirus nephropathy, renal transplantation for less than 2 years, chronic allograft nephropathy, and early acute rejection (EAR, within the first three months).

Acute or chronic rejection based on clinical and laboratory findings was proven by transplant kidney biopsy. Initially pulse steroid therapy was given and if no response was achieved treatment pursued with antithymocyte globulin. The number of acute rejections before and after steroid discontinuation was recorded.

The study protocol was approved by the local ethics committee of the center and is in

accordance with the principles of the Declaration of Helsinki.

Clinical, laboratory and demographic characteristics of the study population including dialysis vintage, duration of transplantation, immunosuppressive and other medications, body mass index, number of HLA mismatches, blood pressure (BP) and late acute rejection episodes

( three months post-transplantation) were recorded.

The uric acid levels before and after steroid discontinuation at an average of $3.9 \pm 2.1$ years and the concurrent creatinine levels and the number of acute rejections were recorded.

Immunosuppressive therapy protocol:

All patients were given basilixumab as induction treatment on Days 0 and 4. Routine immunosuppression consisted of tacrolimus ( 0.1 to $0.2 \mathrm{mg} / \mathrm{kg} /$ day orally divided dose), mycophenolate mofetil (1000 mg orally twice daily), and prednisolone ( $5 \mathrm{mg} /$ day orally).

\section{Statistical analysis:}

Statistical analysis was performed with SPSS software (Statistical Package for the Social Sciences, version 11.5, SSPS Inc, Evanston, Ill, United States). Results were considered as statistically significant if the $P$ value was $<0.05$. Data are shown as the mean $\pm \mathrm{SD}$. Comparisons between the 2 groups were performed by means of the Student $t$ test (Paired Samples test) for normally distributed continuous variables and with the MannWhitney $U$ test for non-normally distributed continuous variables. For comparisons of categorical variables, we used the chi-square test. Cox regression (multiple linear regression) analysis was used to measure the effect of the UA level, acute rejection and HLA mismatch on creatinine.

\section{Results:}


The demographic and clinical characteristics of the patients are shown in Table- 1 . The predominant cause of renal failure was hypertensive nephropathy (primary hypertensive ones) $(\mathrm{n}=35,62.5 \%)$ followed by polycystic kidney disease $(\mathrm{n}=11.2,20.0 \%)$, stone $(\mathrm{n}=5.6,10 \%)$, unknown $(\mathrm{n}=5.3,9.5 \%)$ and glomerulonephritis $(\mathrm{n}=1.1$, $2 \%$ ).

The blood pressure of patients in the primary hypertensive group was under control with $10 \mathrm{mg}$ of amlodipine. There was no statistically significant difference between the blood pressure of the patients using amlodipine before and after steroid discontinuation ( $\mathrm{p}>0.05)$, and uric acid levels did not differ significantly ( $\mathrm{p}>0.05)$. No significant difference was found in uric acid levels between men and women $(\mathrm{p}<0.05)$. No significant relationship was found between age, gender, pre-transplantation dialysis duration, total transplant duration, duration after steroid discontinuation, and body mass index and graft survival $(\mathrm{p}>0.05)$.

However, an increase was observed in uric acid and creatinine levels after the cessation of steroid therapy. A significant correlation was observed between creatinine and uric acid levels $(\mathrm{p}<0.001)$ after steroid discontinuation (Table-2).

HLA mismatches of the patients were evaluated. A statistically significant correlation was found between increased creatinine levels after steroid discontinuation and graft survival with higher HLA mismatch numbers; $39(69.6 \%)$ patients with mismatch of [?]2, and 17 patients with mismatch of $<2(30.4 \%)(\mathrm{p}=0.049)$.

No significant correlation was found between the number of LAR before $(\mathrm{n}=3,5.3 \%)$ and after steroid discontinuation ( $\mathrm{n}=19,33.9 \%)$ and creatinine after steroid discontinuation ( $\mathrm{p}=0.535 \mathrm{p}=0.147$, respectively). Although the number of LAR increased after steroid discontinuation, it was not statistically significant.

Also, predictive risk factors for graft dysfunction, including uric acid and uric acid levels, the number of HLA mismatch, and duration of were analyzed using a multivariate Cox proportional hazards model. The uric acid levels and number of HLA mismatches after discontinuation of steroids were were found to be independent risk factors for graft dysfunction $(\mathrm{p}=0.048$ and $\mathrm{p}=0.044)$.

\section{Discussion:}

In this study, the relationship between hyperuricemia prevalence and graft survival in renal transplant patients using steroids was investigated after steroid discontinuation. The prevalence of hyperuricemia is higher in posttransplant patients compared to the healthy population. ${ }^{10}$ In a study among kidney recipients the prevalence of hyperuricemia was $70 \%$ and $80 \%$ at the baseline and at 2.2 years after transplantation, respectively. ${ }^{3}$ In our study, we observed that the discontinuation of streroids contributed to the increase in uric acid levels. Steroids are known to increase uric acid excretion. ${ }^{9}$ Moreover, posttransplant maintenance immunosuppressive drugs have an effect on uric acid metabolism involving its excretion and absorption. Tacrolimus, for example, increases uric acid levels through reduction of urate excretion. ${ }^{8}$ Initially all patients in the study group used tacrolimus and mycophenolate mofetil and prednisolone as maintenance immunusuppression regimen.

Acute rejection $(\mathrm{AR})$ is a common complication in renal transplantation and is associated with reduced graft survival. ${ }^{3}$ It can occur in less than three months (EAR) or after three months of transplantation (LAR). ${ }^{11}$

AREs had no adverse effect on long-term renal graft function if they were successfully treated. ${ }^{12}$ In the recent years, the incidence of AREs has decreased due to the introduction of newer immunosuppressive agents. ${ }^{13}$ The main factors for a more successful long-term allograft outcome are immunological such as low rate of ARE and advancement of immunosuppressant agents. ${ }^{14,15}$ However, new immunosuppressive agents have a little effect on chronic graft dysfunction and long-term renal allograft survival is still a concern. ${ }^{16}$ Recent research has confirmed that with new immunosuppressive therapy, an EAR is no longer a risk factor of longterm graft failure and chronic allograft nephropathy. ${ }^{17}$ In contrast, another research showed that only LARs have adverse effects on long-term graft function. ${ }^{18}$ Another study showed that EARs were not significant risk factors of chronic graft loss compared with LARs. ${ }^{11}$ In our study, although there was a significant increase in the number of LARs with steroid discontinuation, no effect was found on marrow survival. We concluded 
that LAR treatments are applied effectively to our patients. The discontinuation of the steroid ${ }^{19}$ may not be the only cause, but it may have triggered LAR.

HLA compatibility is one of the best methods to define immunological compatibility between kidney transplant donors and recipients. Certain studies show no statistically

significant difference in graft survival in donor pairs in the case of HLA mismatch ${ }^{20}$, but in other studies, the presence of HLA mismatch negatively affects the development of acute rejection attacks and graft survival. $^{20,21}$ A linear negative relationship was found between HLA mismatch and transplant survival rates. $^{22}$ In our study, a significant negative correlation was found between HLA mismatch and graft survivalstrongly supporting the negative effects of HLA mismatches on the graft as well as the negative effects of high uric acid levels after steroid discontinuation.

Hyperuricemia contributes to kidney dysfunction by several mechanisms. Elevated uric acid levels may cause progression of renal injury by activating the renin-angiotensin system and resulting in increases in both systemic and glomerular pressures, and cyclooxygenase- $2 .{ }^{12,23}$ In this study, we excluded hypertensive patients, and patients with uncontrolled blood pressure on more than one-drug regimen. In another study the effect of serum uric acid level on endothelial damage and inflammation markers in renal allograft recipients was determined. ${ }^{24}$

Gerhardt et al showed that in hyperuricemic patients allograft survival decreased significantly at 2, 4 and 5 years compared to normouricemic patients. ${ }^{25}$ Our study also emphasizes that the elevation of UA, which may have developed with the cessation of steroids, negatively affects the graft functions.

In our study, the low number of normouricemic patients after steroid discontinuation in RT patients (the major limitation of our study) suggested that steroid discontinuation was an additional factor for the occurrence of posttransplant hyperuricemia.

Saying that hyperuricemia alone has an adverse effect on a failing survivor would not be correct. One should keep in mind that subclinical rejection attacks not reflected in the clinic during follow-up may negatively affect graft survival.

In conclusion, according to the model obtained as a result of multivariate linear analysis in our study, hyperuricemia and HLA mismatch counts are independent predictive factors for graft dysfunction in patients with steroid discontinuation. Accordingly, the negative effects of the modeling factors used in our study should be kept in mind in terms of long-term graft survival in patients who are planned to discontinue steroids. While making predictions for graft survival in patients planned to have steroid discontinuation, our study might provide a guidance in this regard; but we wanted to emphasize the need for multi-center studies with more cases, taking into account our limitations.

\section{LIMITATIONS:}

We screened 678 patients who stopped steroids in our center between 1999-2020 $(\mathrm{n}=56)$. Highly selected cases were included in the study, keeping the admission criteria comprehensive. However, not comparing these two groups in terms of graft survival is the major limitation of the study, since the number of patients with steroid discontinuation and normal uric acid levels was very low.

Confirming the role of UA in progressive renal disease as well as in cardiovascular disease in the transplant population will necessitate larger sample sizes to allow for the adjustment of confounding variables, as well as clinical trials of maneuvers for altering UA levels. Until such studies can be realized, hyperuricemia remains a promising marker for progressive renal allograft dysfunction and cardiovascular disease after renal transplantation.

Conflict of Interest Statement: The authors declared no conflict of interest.

Funding: There is no funding related to this article. 
Authors' contributions: Hulya Colak, Sibel Ersan, Banu Yilmaz, Mehmet Tanrisev collected the information, reviewed the literature, and wrote the manuscript. Zumrut Unal critically reviewed the manuscript and İsmail Sert approved the final form. All authors read and approved the final manuscript. Hülya Çolak and Zümrüt Ünal performed data analysis, statistics and interpretation. Funding secured by Hulya Çolak.

\section{RESOURCES:}

1- Richard J Johnson, George L Bakris, Claudio Borghi, Michel B Chonchol, David Feldman, Miguel A Lanaspa, Tony R Merriman, Orson W Moe, David B Mount, Laura Gabriella Sanchez Lozada, Eli Stahl, Daniel E Weiner, Glenn M Chertow. Hyperuricemia, Acute and Chronic Kidney Disease, Hypertension, and Cardiovascular Disease: Report of a Scientific Workshop Organized by the National Kidney Foundation. Am J Kidney Dis

. 2018 Jun;71(6):851-865doi: 10.1053/j.ajkd.2017.12.009. Epub 2018 Feb 27.

2- Johnson RJ, Kang DH, Feig D, Kivlighn S, Kanellis J, Watanabe S, Tuttle KR, Rodriguez-Iturbe B,

Herrera-Acosta J, Mazzali M. Is there a pathogenetic role for uric acid in hypertension and cardiovascular and renal disease? Hypertension 2003;41:1183-1190. doi: 10.1161/01.HYP.0000069700.62727.C5. Epub 2003 Apr 21.

3- Armstrong KA, Johnson DW, Campbell SB, Isbel NM, Hawley CM. Does uric acid have a pathogenetic role in graft dysfunction and hypertension in renal transplant recipients? Transplantation 2005;80:15651571.PMID: 16371927 DOI: 10.1097/01.tp.0000183895.88572.13

4-Mazzali M. Uric acid and transplantation. Semin Nephrol 2005;25:505.PMID: 15660335 DOI: 10.1016/j.semnephrol.2004.09.008

5- Borghi C, Rosei EA, Bardin T, Dawson J, Dominiczak A, Kielstein JT, Manolis AJ, Perez-Ruiz F, Mancia G.J Hypertens. 2015 Sep;33(9):1729-41; discussion 1741. doi: 10.1097/HJH.0000000000000701).

6-Viazzi F, Leoncini G, Pontremoli R. Cardiovascular and renal effects of hyperuricaemia and gout.Reumatismo. 2012 Jan 19;63(4):253-62. doi: 10.4081. PMID: 22303532 DOI: 10.4081/reumatismo.2011.253

7- Farah Bandukwala, Michael Huang, Jeffrey S Zaltzman, Michelle M Nash, G V Ramesh PrasadAssociation of uric acid with inflammation, progressive renal allograft dysfunction and post-transplant cardiovascular risk. Am J Cardiol.

2009 Mar 15;103(6):867-71. doi:10.1016/j.amjcard. 2008.11.042. Epub 2009 Jan 24. 8- Kanbay M, Akcay A, Huddam B et al. Influence of cyclosporine and tacrolimus on serum uric acid levels in stable kidney transplant recipients. Transplant Proc. 2005;37:311920. PMID: 16213325 DOI: 10.1016/j.transproceed.2005.08.042

Dujovne CA, Azarnoff DL: Clinical complications of corticosteroid therapy: A selected review. Steroid therapy. Med Clin North Am. 1973 Sep;57(5):1331-42. DOI: 10.1016/s0025-7125(16)32233-7.

Akgul A, Bilgic A, Ibis A, Ozdemir FN, Arat Z, Haberal M. Is uric acid a predictive factor for graft dysfunction in renal transplant recipients? Transplant Proc. 2007 May;39(4):1023-6. PMID: 17524881 DOI: $10.1016 / j$.transproceed.2007.03.028

11-Jalalzadeh M, Mousavinasab N, Peyrovi S, Ghadiani MH. The impact of acute rejection in kidney transplantation on long-term allograft and patient outcome. Nephrourol Mon. 2015 Jan 20;7(1):e24439. PMID: 25738128 PMCID: PMC4330708DOI: 10.5812/numonthly.24439.

12- Burke G, 3rd., Ciancio G, Figueiro J, Olson L, Gomez C, Rosen A, et al. Can acute rejection be prevented in SPK transplantation? Transplant Proc. 2002;34(5):1913-4. PMID: 12176626 DOI: 10.1016/s0041$1345(02) 03149-4$. 
13-Hamida FB, Barbouch S, Bardi R, Helal I, Kaaroud H, Fatma LB, et al. Acute rejection episodes after kidney transplantation. Saudi J Kidney Dis Transpl. 2009;20(3):370-4. PMID: 19414936

14- Wang D, Xu TZ, Chen JH, Wu WZ, Yang SL, Lin WH, et al. Factors influencing second renal allograft survival: a single center experience in China. Transpl Immunol. 2009;20(3):150-4. PMID: 18848988 DOI: $10.1016 /$ j.trim.2008.09.010

15- Kuypers DR. Immunosuppressive drug therapy and subclinical acute renal allograft rejection: impact and effect. Transplantation. 2008;85(7 Suppl):S25-30. PMID: 18401259 DOI: 10.1097/TP.0b013e318169c48d

16- Pascual M, Theruvath T, Kawai T, Tolkoff-Rubin N, Cosimi AB. Strategies to improve long-term outcomes after renal transplantation. N Engl J Med. 2002;346(8):580-90. PMID: 11856798 DOI: 10.1056/NEJMra011295

17- Pallardo Mateu LM, Sancho Calabuig A, Capdevila Plaza L, Franco Esteve A. Acute rejection and late renal transplant failure: risk factors and prognosis. Nephrol Dial Transplant. 2004;19 Suppl 3:iii38-42. PMID: 15192134DOI: $10.1093 / n d t / g f h 1013$

18- Meier-Kriesche HU, Steffen BJ, Hochberg AM, Gordon RD, Liebman MN, Morris JA, et al. Long-Term Use of Mycophenolate Mofetil is Associated With a Reduction in the Incidence and Risk of Late Rejection. Am J Transplant.2003;3(1):68-73. PMID: 12492713 DOI: 10.1034/j.1600-6143.2003.30112.x

Tönshoff B. Immunosuppressants in Organ Transplantation. Handb Exp Pharmacol. 2020;261:441-469. PMID: 31820175 DOI: 10.1007/164_2019_331

20-Eun Jeong Ko, Ji Hyun Yu, Chul Woo Yang, Byung Ha Chung. Korean Organ Transplantation Registry Study Group outcomes of ABO- and HLA-incompatible kidney transplantation: a nationwide cohort study. Transpl Int, 30 (2017), s. 1215-1225. PMID: 28493630 DOI: 10.1111/tri.12979

21-J. Lee, D.G. Kim, B.S. Kim, M.S. Kim, S. Il Kim, Y.S. Kim, vdLee J, Kim DG, Kim BS, Kim MS, Il Kim S, Kim YS, et al. Early hospital readmissions after ABO- or HLA- incompatibleliving donor kidney transplantation. Sci Rep 2019;9:3246. PMID: 30824777 PMCID: PMC6397202DOI: 10.1038/s41598-01939841-8

22-Rabi Yacoub, Girish N Nadkarni, Paolo Cravedi, John Cijiang He, Veronica B Delaney, Rebecca Kent, Kinsuk N Chauhan, Steven G Coca, Sander S Florman, Peter S Heeger, Barbara Murphy, Madhav C Menon . Analysis of OPTN/UNOS registry suggests the number of HLA matches and not mismatches is a stronger independent predictor of kidney transplant survival Kidney Int. 2018; Feb;93(2):482-490. doi: 10.1016/j.kint.2017.07.016. Epub 2017 Sep 29. PMID: 28965746 DOI: 10.1016/j.kint.2017.07.016

23-Kang DH, Nakagawa T. Uric acid and chronic renal disease: possible implication of hyperuricemia on progression of renaldis ease. Semin Nephrol. 2005;25:43. PMID: 15660334 DOI: $10.1016 / j . s e m n e p h r o l .2004 .10 .001$

24-Farah Bandukwala 1, Michael Huang, Jeffrey S Zaltzman, Michelle M Nash, G V Ramesh Prasad. Association of uric acid with inflammation, progressive renal allograft dysfunction and post-transplant cardiovascular risk. Am J Cardiol

. 2009 Mar 15;103(6):867-71.doi: 10.1016/j.amjcard.2008.11.042. Epub 2009 Jan 24..

25-Gerhardt U, Grosse HM, Hohage H. Influence of hyperglycemia and hyperuricemia on long- term transplant survival in kidney transplant recipients. Clin Transplant 13:375, 1999. PMID: 10515217 DOI: $10.1034 / j .1399-0012.1999 .130502$

\section{Hosted file}

Table 1.pdf available at https://authorea.com/users/412564/articles/521224-the-relationshipbetween-uric-acid-levels-and-graft-function-in-renal-transplant-patients-who-

discontinued-steroid-therapy 


\section{Hosted file}

table 2.pdf available at https://authorea.com/users/412564/articles/521224-the-relationshipbetween-uric-acid-levels-and-graft-function-in-renal-transplant-patients-whodiscontinued-steroid-therapy 